\title{
Variable Annuity with GMWB: surrender or not, that is the question
}

\author{
$\underline{\text { X. Luo }}^{a}$ and P.V. Shevchenko ${ }^{a}$ \\ ${ }^{\text {a } C S I R O ~ R i s k ~ A n a l y t i c s ~ G r o u p, ~ A u s t r a l i a ~}$ \\ This version: 31 July, 2015 \\ Email: Xiaolin.Luo@csiro.au
}

\begin{abstract}
A variable annuity contract with Guaranteed Minimum Withdrawal Benefit (GMWB) promises to return the entire initial investment through cash withdrawals during the policy life plus the remaining account balance at maturity, regardless of the portfolio performance. We assume that market is complete in financial risk and also there is no mortality risk (in the event of policyholder death, the contract is maintained by beneficiary), thus the annuity price can be expressed as an appropriate expectation. Under the optimal withdrawal strategy of a policyholder, the pricing of variable annuities with GMWB is an optimal stochastic control problem. The surrender feature available in marketed products allows termination of the contract before maturity, making it also an optimal stopping problem.
\end{abstract}

Although the surrender feature is quite common in variable annuity contracts, there appears to be no published analysis and results for this feature in GMWB under optimal policyholder behavior - results found in the literature so far are consistent with the absence of such a feature. Recently, Azimzadeh and Forsyth (2014) prove the existence of an optimal bang-bang control for a Guaranteed Lifelong Withdrawal Benefits (GLWB) contract. In particular, they find that the holder of a GLWB can maximize a writers losses by only ever performing nonwithdrawal, withdrawal at exactly the contract rate, or full surrender. This dramatically reduces the optimal strategy space. However, they also demonstrate that the related GMWB contract is not convexity preserving, and hence does not satisfy the bang-bang principle other than in certain degenerate cases. For GMWB under optimal withdrawal assumption, the numerical algorithms developed by Dai et al. (2008), Chen and Forsyth (2008) and Luo and Shevchenko (2015a) appear to be the only ones found in the literature, but none of them actually performed calculations with surrender option on top of optimal withdrawal strategy. Also, it is of practical interest to see how the much simpler bang-bang strategy, although not optimal for GMWB, compares with optimal GMWB strategy with surrender option.

Recently, in Luo and Shevchenko (2015a), we have developed a new efficient numerical algorithm for pricing GMWB contracts in the case when transition density of the underlying asset between withdrawal dates or its moments are known. This algorithm relies on computing the expected contract value through a high order Gauss-Hermite quadrature applied on a cubic spline interpolation and much faster than the standard partial differential equation methods. In this paper we extend our algorithm to include surrender option in GMWB and compare prices under different policyholder strategies: optimal, static and bang-bang. Results indicate that following a simple but sub-optimal bang-bang strategy does not lead to significant reduction in the price or equivalently in the fee, in comparison with the optimal strategy. We also observed that the extra value added by the surrender option strongly depends on volatility and the penalty charge, among other factors such as contractual rate, maturity and interest rate etc. At high volatility or at low penalty charge, the surrender feature adds very significant value to the GMWB contract - the required fair fee is more than doubled in some cases; thus it is critical to account for surrender feature in pricing of real products. We also performed calculations for static withdrawal with surrender option, which is the same as bang-bang minus the "no-withdrawal" choice. We find that the fee for such contract is only less than $1 \%$ smaller when compared to the case of bang-bang strategy, meaning that the "no-withdrawal" option adds little value to the contract.

Keywords: Variable annuity, optimal stochastic control, optimal stopping time, bang-bang control, Guaranteed Minimum Withdrawal Benefit 


\section{INTRODUCTION}

The world population is becoming older fast. As a consequence the age-related spending is projected to rise dramatically in the coming decades in all the developed countries. Increasingly governments in the developed world realize they cannot afford paying sufficient public pensions and are looking for innovations in retirement income product market. In this paper we consider a variable annuity contract with Guaranteed Minimum Withdrawal Benefit (GMWB) with option to surrender the contract before maturity. This contract promises to return the entire initial investment through cash withdrawals during the policy life plus the remaining account balance at maturity, regardless of the portfolio performance. Thus even when the account of the policyholder falls to zero before maturity, GMWB feature will continue to provide the guaranteed cashflows. In addition, we allow the option to surrender the contract before the maturity which is a standard feature of real products on the market. GMWB allows the policyholder to withdraw funds below or at contractual rate without penalty and above the contractual rate with some penalty. If the policyholder behaves passively and the withdraw amount at each withdrawal date is predetermined at the beginning of the contract, then the behavior of the policyholder is called "static". In this case the paths of the account can be simulated and a standard Monte Carlo simulation method can be used to price the GMWB. On the other hand if the policyholder optimally decide the amount of withdraw at each withdrawal date, then the behavior of the policyholder is called "dynamic". Under the optimal withdrawal strategy of a policyholder, the pricing of variable annuities with GMWB becomes an optimal stochastic control problem; and adding surrender feature makes it also an optimal stopping problem.

The variable annuities with GMWB feature under dynamic and static withdrawal strategies have been considered in a number of papers over the last decade, e.g. Milevsky and Salisbury (2006), Bauer et al. (2008), Dai et al. (2008), Huang and Forsyth (2012); Huang and Kwok (2014), Bacinello et al. (2011). Recently, Azimzadeh and Forsyth (2014) prove the existence of an optimal bang-bang control for a Guaranteed Lifelong Withdrawal Benefits (GLWB) contract. In particular, they find that the holder of a GLWB can maximize a contract writer loss by only ever performing non-withdrawal, withdrawal at exactly the contract rate, or full surrender. This dramatically reduces the optimal strategy space. However, they also demonstrate that the related GMWB contract is not convexity preserving, and hence does not satisfy the bang-bang principle other than in certain degenerate cases. For GMWB under optimal withdrawal assumption, the numerical algorithms developed by Dai et al. (2008) and Chen and Forsyth (2008) appear to be the only ones found in the literature, and both are based on solving corresponding partial differential equation (PDE) via finite difference method.

In the case when transition density of the underlying wealth process between withdrawal dates or its moments are known in closed form, often it can be more convenient and more efficient to utilize direct integration methods to calculate the required annuity value expectations in backward time-stepping procedure. Such an algorithm was developed in Luo and Shevchenko (2014, 2015a) for solving optimal stochastic control problem in pricing GMWB variable annuity. This allows to get virtually instant results for typical GMWB annuity prices on the standard desktop PC. In this paper we adopt this algorithm to price variable annuities with GMWB with surrender option under static, dynamic, and simplified bang-bang withdrawal strategies. To our best knowledge, there are no publications presenting results for GMWB with both optimal withdrawal and surrender features.

In the next section we describe the GMWB product with discrete withdrawals, the underlying stochastic model and the optimization problem. Section 3 describes numerical algorithm utilized for pricing In Section 4, numerical results for the fair fees under a series GMWB contract conditions are presented. Concluding remarks are given in Section 5.

\section{MODEL}

We assume that market is complete in financial risk and also there is no mortality risk (in the event of policyholder death, the contract is maintained by beneficiary), thus the annuity price can be expressed as expectation under the risk neutral process of underlying asset. Let $S(t)$ denote the value of the reference portfolio of assets (mutual fund index, etc.) underlying the variable annuity policy at time $t$ that under no-arbitrage condition follows the risk neutral stochastic process

$$
d S(t)=r(t) S(t) d t+\sigma(t) S(t) d B(t)
$$

where $B(t)$ is the standard Wiener process, $r(t)$ is risk free interest rate and $\sigma(t)$ is volatility. For simplicity hereafter we assume that model parameters are piece-wise constant functions of time for time discretization $0=t_{0}<t_{1}<\cdots<t_{N}=T$, where $t_{0}=0$ is today and $T$ is annuity contract maturity. Denote correspond- 
ing asset values as $S\left(t_{0}\right), \ldots, S\left(t_{N}\right)$; and risk-free interest rate and volatility as $r_{1}, \ldots, r_{N}$ and $\sigma_{1}, \ldots, \sigma_{N}$ respectively. That is, $r_{1}$ is the interest rate for time teriod $\left(t_{0}, t_{1}\right] ; r_{2}$ is for $\left(t_{1} ; t_{2}\right]$, etc and similar for volatility.

The premium paid by policyholder upfront at $t_{0}$ is invested into the reference portfolio of risky assets $S(t)$. Denote the value of this variable annuity account (hereafter referred to as wealth account) at time $t$ as $W(t)$, i.e. the upfront premium paid by policyholder is $W(0)$. GMWB guarantees the return of the premium via withdrawals $\gamma_{n} \geq 0$ allowed at times $t_{n}, n=1, \ldots, N$. Let $N_{w}$ denote the number of withdrawals in a year (e.g. $N_{w}=12$ for a monthly withdrawal), then the total number of withdrawals $N=\left\lceil N_{w} \times T\right\rceil$. The total of withdrawals cannot exceed the guarantee $W(0)$ and withdrawals can be different from contractual (guaranteed) withdrawal $G_{n}=W(0)\left(t_{n}-t_{n-1}\right) / T$, with penalties imposed if $\gamma_{n}>G_{n}$. Denote the annual contractual rate as $g=1 / T$.

Denote the value of the guarantee at time $t$ as $A(t)$, hereafter referred to as guarantee account. Obviously, $A(0)=W(0)$. For clarity of notation, denote the time immediately before $t$ (i.e. before withdrawal) as $t^{-}$, and immediately after $t$ (i.e. after withdrawal) as $t^{+}$. Then the guarantee balance evolves as

$$
A\left(t_{n}^{+}\right)=A\left(t_{n}^{-}\right)-\gamma_{n}=A\left(t_{n-1}^{+}\right)-\gamma_{n}, \quad n=1,2, \ldots, N
$$

with $A\left(T^{+}\right)=0$, i.e. $W(0)=A(0) \geq \gamma_{1}+\cdots+\gamma_{N}$ and $A\left(t_{n-1}^{+}\right) \geq \sum_{k=n}^{N} \gamma_{k}$. The account balance $A(t)$ remains unchanged within the interval $\left(t_{n-1}, t_{n}\right), n=1,2, \ldots, N$.

In the case of reference portfolio process (1), the wealth account $W(t)$ evolves as

$$
\begin{aligned}
W\left(t_{n}^{-}\right) & =\frac{W\left(t_{n-1}^{+}\right)}{S\left(t_{n-1}\right)} S\left(t_{n}\right) e^{-\alpha d t_{n}}=W\left(t_{n-1}^{+}\right) e^{\left(r_{n}-\alpha-\frac{1}{2} \sigma_{n}^{2}\right) d t_{n}+\sigma_{n} \sqrt{d t_{n}} z_{n}}, \\
W\left(t_{n}^{+}\right) & =\max \left(W\left(t_{n}^{-}\right)-\gamma_{n}, 0\right), n=1,2, \ldots, N,
\end{aligned}
$$

where $d t_{n}=t_{n}-t_{n-1}, z_{n}$ are iid standard Normal random variables and $\alpha$ is the annual fee charged by the insurance company. If the account balance becomes zero or negative, then it will stay zero till maturity.

The cashflow received by the policyholder at withdrawal time $t_{n}$ is given by

$$
C_{n}\left(\gamma_{n}\right)= \begin{cases}\gamma_{n}, & \text { if } 0 \leq \gamma_{n} \leq G_{n}, \\ G_{n}+(1-\beta)\left(\gamma_{n}-G_{n}\right), & \text { if } \gamma_{n}>G_{n},\end{cases}
$$

where $G_{n}$ is contractual withdrawal. That is, penalty is applied if withdrawal $\gamma_{n}$ exceeds $G_{n}$, i.e. $\beta \in[0,1]$ is the penalty applied to the portion of withdrawal above $G_{n}$.

If the policyholder decides to surrender at time slice $\tau \in(1, \ldots, N-1)$, then policyholder receives cashflow $D_{\tau}\left(W\left(t_{\tau}\right), A\left(t_{\tau}\right)\right)$ and contract stops. For numerical example we assume that

$$
D_{\tau}\left(W\left(t_{\tau}\right), A\left(t_{\tau}\right)\right):=C_{\tau}\left(\max \left(W\left(t_{\tau}\right), A\left(t_{\tau}\right)\right)\right)
$$

other standard surrender conditions can easily be implemented.

Denote the value of variable annuity at time $t$ as $Q_{t}(W(t), A(t))$, i.e. it is determined by values of the wealth and guarantee accounts $W(t)$ and $A(t)$. At maturity, if not surrendered earlier, the policyholder takes the maximum between the remaining guarantee withdrawal net of penalty charge and the remaining balance of the personal account, i.e. the final payoff is

$$
Q_{t_{N}^{-}}\left(W\left(T^{-}\right), A\left(T^{-}\right)\right):=h_{N}\left(W\left(T^{-}\right), A\left(T^{-}\right)\right)=\max \left(W\left(T^{-}\right), C_{N}\left(A\left(T^{-}\right)\right)\right) .
$$

Under the above assumptions/conditions, the fair no-arbitrage value of the annuity at time $t_{0}$ is

$$
\begin{aligned}
& Q_{t_{0}}\left(W\left(t_{0}\right), A\left(t_{0}\right)\right)=\max _{\tau, \gamma_{1}, \ldots, \gamma_{\widetilde{N}}} \mathrm{E}_{t_{0}}\left[B(0, \tau) D_{\tau}\left(W\left(t_{\tau}^{-}\right), A\left(t_{\tau}^{-}\right)\right) \mathbb{I}_{\left\{t_{\tau}<T\right\}}\right. \\
& \left.\quad+B(0, N) h_{N}\left(W\left(T^{-}\right), A\left(T^{-}\right)\right)\left(1-\mathbb{I}_{\left\{t_{\tau}<T\right\}}\right)+\sum_{j=1}^{\widetilde{N}} B(0, j) C_{j}\left(\gamma_{j}\right)\right], \quad \widetilde{N}=\min (\tau, N)-1
\end{aligned}
$$

where $B_{0, n}=\exp \left(-\int_{0}^{t_{n}} r(\tau) d \tau\right)$ is discounting factor and $\mathbb{I}_{\{\cdot\}}$ is indicator function. Note that the today's value of the annuity policy $Q_{0}(W(0), A(0))$ is a function of policy fee $\alpha$. Here, $\tau$ is stopping time and 
$\gamma_{1}, \ldots, \gamma_{N-1}$ are the control variables chosen to maximize the expected value of discounted cashflows, and expectation $\mathrm{E}_{0}[\cdot]$ is taken under the risk-neutral process conditional on $W_{0}$ and $A_{0}$. The fair fee value of $\alpha$ corresponds to $Q_{0}(W(0), A(0))=W(0)$. It is important to note that control variables and stopping time can be different for different realizations of underlying process and moreover the control variable $\gamma_{n}$ affects the transition law of the underlying wealth process from $t_{n}$ to $t_{n+1}$. Overall, evaluating GMWB with surrender feature is solving optimal stochastic control problem with optimal stopping.

Denote the state vector at time $t_{n}$ as $X_{n}=\left(W\left(t_{n}^{-}\right), A\left(t_{n}^{-}\right)\right)$. Given that $\boldsymbol{X}=\left(X_{1}, \ldots, X_{N}\right)$ is Markov process, it is easy to recognize that the annuity valuation under the optimal withdrawal strategy (8) is optimal stochastic control problem for Markov process that can be solved recursively to find annuity value $Q_{t_{n}}(x)$ at $t_{n}, n=N-1, \ldots, 0$ via backward induction

$$
Q_{t_{n}}(x)=\max \left(\sup _{0 \leq \gamma_{n} \leq A\left(t_{n}^{-}\right)}\left(C_{n}\left(\gamma_{n}\left(X_{n}\right)\right)+e^{-r_{n+1} d t_{n+1}} \int Q_{t_{n+1}}\left(x^{\prime}\right) K_{t_{n}}\left(d x^{\prime} \mid x, \gamma_{n}\right)\right), D_{n}(x)\right)
$$

starting from final condition $Q_{T}(x)=\max \left(W\left(T^{-}\right), C_{N}\left(A\left(T^{-}\right)\right)\right)$. Here $K_{t_{n}}\left(d x^{\prime} \mid x, \gamma_{n}\right)$ is the stochastic kernel representing probability to reach state in $d x^{\prime}$ at time $t_{n+1}$ if the withdrawal (action) $\gamma_{n}$ is applied in the state $x$ at time $t_{n}$. For a good textbook treatment of stochastic control problem in finance, see Bäuerle and Rieder (2011). Explicitly, this backward recursion can be solved as follows.

The annuity price at any time $t$ for a fixed $A(t)$ is a function of $W$ only. Note $A\left(t_{n-1}^{+}\right)=A\left(t_{n}^{-}\right)=A$ is constant in the period $\left(t_{n-1}^{+}, t_{n}^{-}\right)$. Thus in a backward time-stepping setting (similar to a finite difference scheme) the annuity value at time $t=t_{n-1}^{+}$can be evaluated as the following expectation

$$
Q_{t_{n-1}^{+}}\left(W\left(t_{n-1}^{+}\right), A\right)=\mathrm{E}_{t_{n-1}}\left[e^{-r_{n} d t_{n}} Q_{t_{n}^{-}}\left(W\left(t_{n}^{-}\right), A\right) \mid W\left(t_{n-1}^{+}\right), A\right] .
$$

Assuming the conditional probability distribution density of $W\left(t_{n}^{-}\right)$given $W\left(t_{n-1}^{+}\right)$is known as $p_{n}\left(w\left(t_{n}\right) \mid w\left(t_{n-1}\right)\right)$, then the above expectation can be evaluated by

$$
Q_{t_{n-1}^{+}}\left(W\left(t_{n-1}^{+}\right), A\right)=\int_{0}^{+\infty} e^{-r_{n} d t_{n}} p_{n}\left(w \mid W\left(t_{n-1}^{+}\right)\right) Q_{t_{n}^{-}}(w, A) d w .
$$

In the case of wealth process (3) the transition density $p_{n}\left(w\left(t_{n}\right) \mid w\left(t_{n-1}\right)\right)$ is known in closed form and we will use Gauss-Hermite quadrature for the evaluation of the above integration over an infinite domain. The required continuous function $Q_{t}(W, A)$ will be approximated by a cubic spline interpolation on a discretized grid in the $W$ space.

Any change of $A(t)$ only occurs at withdrawal dates. After the amount $\gamma_{n}$ is drawn at $t_{n}$, the wealth account reduces from $W\left(t_{n}^{-}\right)$to $W\left(t_{n}^{+}\right)=\max \left(W\left(t_{n}^{-}\right)-\gamma_{n}, 0\right)$, and the guarantee balance drops from $A\left(t_{n}^{-}\right)$to $A\left(t_{n}^{+}\right)=A\left(t_{n}^{-}\right)-\gamma_{n}$. Thus the jump condition of $Q_{t}(W, A)$ across $t_{n}$ is given by

$$
\begin{aligned}
& Q_{t_{n}^{-}}\left(W\left(t_{n}^{-}\right), A\left(t_{n}^{-}\right)\right) \\
& =\max \left(\max _{0 \leq \gamma_{n} \leq A\left(t_{n}^{-}\right)}\left[Q_{t_{n}^{+}}\left(\max \left(W\left(t_{n}^{-}\right)-\gamma_{n}, 0\right), A\left(t_{n}^{-}\right)-\gamma_{n}\right)+C_{n}\left(\gamma_{n}\right)\right], D_{n}\left(W\left(t_{n}^{-}\right), A\left(t_{n}^{-}\right)\right)\right) .
\end{aligned}
$$

For optimal strategy, we chose a value for $\gamma_{n}$ under the restriction $0 \leq \gamma_{n} \leq A\left(t_{n}^{-}\right)$to maximize the function value $Q_{t_{n}^{-}}(W, A)$ in (12). Repeatedly applying (11) and (12) backwards in time starting from

$$
Q_{t_{N}^{-}}\left(W\left(T^{-}\right), A\left(T^{-}\right)\right)=\max \left(W\left(T^{-}\right), C_{N}\left(A\left(T^{-}\right)\right)\right)
$$

gives us annuity value at $t=0$.

In additional to dynamic and static strategies, in this paper we also consider bang-bang strategy which is simplified sup-optimal strategy where the policy holder at each $t_{n}$ can either make withdrawal at contractual rate $G_{n}$, make no withdrawal or surrender.

\section{Numerical ALgORITHM}

A very detailed description of the algorithm that we adapt for pricing GMWB with surrender can be found in Luo and Shevchenko (2015a). Below we outline the main steps. We discretize the asset domain $\left[W_{\min }, W_{\max }\right]$ 
by $W_{\min }=W_{0}<W_{1}, \ldots, W_{M}=W_{\max }$, where $W_{\min }$ and $W_{\max }$ are the lower and upper boundary, respectively. The idea is to find annuity values at all these grid points at each time step from $t_{n}^{-}$to $t_{n-1}^{+}$ through integration (11), starting at maturity $t=t_{N}^{-}=T^{-}$. At each time step we evaluate the integration (11) for every grid point by a high accuracy Gauss-Hermite numerical quadrature; it can also be accomplished by solving corresponding PDE using finite difference method that we implemented for benchmarking. At time step $t_{n}^{-} \rightarrow t_{n-1}^{+}$, the annuity value at $t=t_{n}^{-}$is known only at grid points $W_{m}, m=0,1, \ldots, M$. In order to approximate the continuous function $Q_{t}(W, A)$ from the values at the discrete grid points, we use the cubic spline interpolation which is smooth in the first derivative and continuous in the second derivative.

For guarantee account balance variable $A$, we introduce an auxiliary finite grid $0=A_{1}<\cdots<A_{J}=W(0)$ to track the remaining guarantee balance $A$, where $J$ is the total number of nodes in the guarantee balance amount coordinate. For each $A_{j}$, we associate a continuous solution $Q_{t}\left(W, A_{j}\right)$. At every jump we let $A$ to be one of the grid points $A_{j}, 1 \leq j \leq J$. For any $W=W_{m}, m=0,1, \ldots, M$ and $A=A_{j}, j=1, \ldots, J$, given that withdrawal amount can only take the pre-defined values $\gamma=A_{j}-A_{k}, k=1,2, \ldots, j$, irrespective of time $t_{n}$ and account value $W_{m}$, the jump condition (12) takes the following form for the specific numerical setting

$$
Q_{t_{n}^{-}}\left(W_{m}, A_{j}\right)=\max \left(\max _{1 \leq k \leq j}\left[Q_{t_{n}^{+}}\left(\max \left(W_{m}-A_{j}+A_{k}, 0\right), A_{k}\right)+C_{n}\left(A_{j}-A_{k}\right)\right], D_{n}\left(W_{m}, A_{j}\right)\right) .
$$

Overall we have $J$ numerical solutions (obtained through integration) to track, corresponding to each of the $A_{j}$ value, $1 \leq j \leq J$. Stepping backward in time, we find $Q_{0}(W(0), A(0))$ that depends on the policy fee $\alpha$. Finally, we calculate fair fee value of $\alpha$ corresponding to $Q_{0}(W(0), A(0))=W(0)$ that obviously requires iterative process.

\section{Numerical Results}

Below we present numerical results for fair fee of GMWB with surrender option under optimal and suboptimal bang-bang withdrawal strategies. For convenience we denote results for optimal withdrawal strategy without surrender option as GMWB, and with surrender option as GMWB-S. As discussed in Luo and Shevchenko (2015a), only very few results for GMWB under dynamic policyholder behavior can be found in the literature, and these results are for GMWB without the surrender option. For validation purposes, perhaps the most accurate results are found in Chen and Forsyth (2008), which were obtained with a very fine mesh in a detailed convergence study. As shown in Table 1, our GMWB results for fair fee compare very well with those of Chen and Forsyth (2008). The maximum absolute difference in the fair fee rate between the two numerical studies is only 0.3 basis point (a basis point is $0.01 \%$ of a rate).

Table 1 shows some very interesting comparison among GMWB, GMWB-S and bang-bang results. At volatility $\sigma=0.2$, the fair fee for GMWB-S is virtually the same as GMWB, meaning surrender adds little value to the optimal strategy; at high volatility $\sigma=0.3$, fees for GMWB-S are significantly higher than GMWB, up to $50 \%$ higher at the half-yearly frequency. This may suggest that at high volatility it is optimal to surrender at high values of account balance or guarantee level. In addition, it also shows higher frequency gives higher extra value to the surrender option. Comparing bang bang with GMWB-S, the fees are below the optimal strategy as expected, but the values are not very significantly lower at both volatility values - it is only about $10 \%$ lower at most.

Figure 1 shows curves of the fee as a function of the contractual annual withdraw rate, given $\sigma=0.2, r=0.05$ and $\beta=0.1$. It compares four cases: static (without surrender), GMWB, GMWB-S and bang-bang, all at quarterly withdrawal frequency with $10 \%$ penalty charge, i.e. $\beta=0.1$. This comparison also shows GMWB$\mathrm{S}$ and GMWB have virtually the same fees at $\sigma=0.2$, and bang-bnag is only slightly below GMWB-S, confirming results in Table 1 . However, at the same volatility $\sigma=0.2$, new features emerge if we reduce the penalty charge from $\beta=0.1$ to $\beta=0.05$, as shown in Figure 2 . When the penalty charge is reduced and all other parameters are unchanged, the surrender option adds more significant value to GMWB - in fact the fees are more than doubled at low to moderate contractual withdraw rate (or equivalently long or moderate maturity), i.e. fees for GMWB-S are more than twice as those for GMWB. With reduced penalty, fees for bang-bang are still close to the optimal strategy with surrender option, the GMWB-S.

We also performed calculations for static withdrawal with surrender option, which is the same as bang-bang minus the "no-withdrawal" choice. We find the fee for such contract is only less than $1 \%$ smaller than the bang-bang strategy, meaning the "no-withdrawal" option adds little value to the contract. Finally, different penalty functions can be applied to the surrender (i.e. surrender cashflow can be different from (6)). For 
example, instead of penalizing only the amount exceeding the contractual withdrawal rate, we can penalize the entire termination amount. In this case we find both GMWB-S and bang-bang yield only slightly lower fees for a given $\beta$ - this is perhaps not very surprising since when it is optimal to surrender, the amount must be much higher than the contractual rate, thus penalizing the entire amount is not much more severe than penalizing only the exceeded part.

Table 1. Comparison of fair fee $\alpha$ in basis points (a basis point is $0.01 \%$ ) between results of GMWB, GMWB$\mathrm{S}$ and bang-bang. Results under "Chen \& Forsyth" are for GMWB. The input parameters are $g=10 \%$, $\beta=10 \%, r=5 \%$ and $\sigma=0.2$. The withdrawal frequency is quarterly.

\begin{tabular}{cccccc}
\hline frequency & volatility & Chen \& Forsyth & GMWB & GMWB-S & Bang Bang \\
\hline yearly & 0.2 & 129.1 & 129.1 & 129.2 & 123.9 \\
half-yearly & 0.2 & 133.5 & 133.7 & 134.0 & 125.6 \\
yearly & 0.3 & 293.3 & 293.5 & 418.4 & 392.9 \\
half-yearly & 0.3 & 302.4 & 302.7 & 456.5 & 410.7 \\
\hline
\end{tabular}

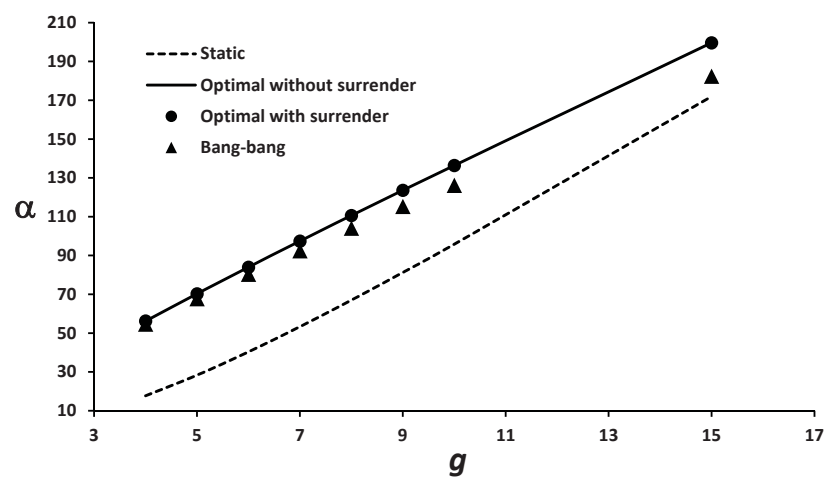

Figure 1. Fair fee $\alpha$ as a function of annual guarantee rate $g$ for static, GMWB, GMWB-S and bang-bang at a quarterly withdraw rate. The fixed input parameters are $\beta=10 \%, r=5 \%$ and $\sigma=0.2$.

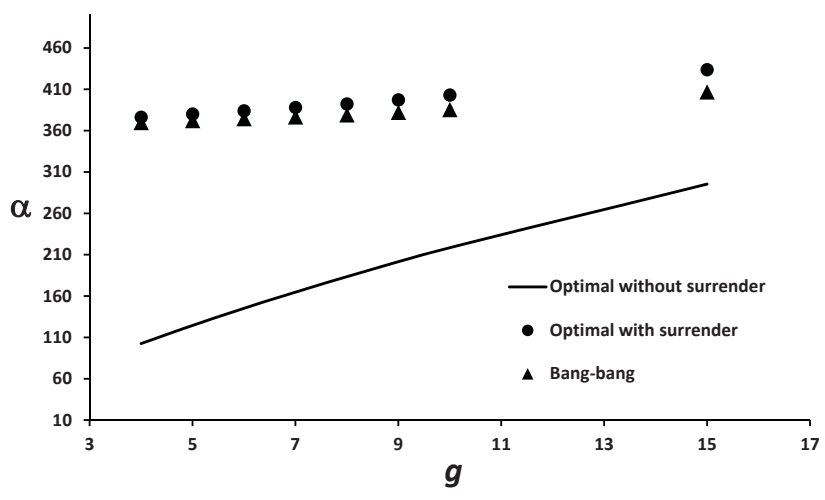

Figure 2. Fair fee $\alpha$ as a function of annual guarantee rate $g$ for static, GMWB, GMWB-S and bang-bang at a quarterly withdraw rate. The fixed input parameters are $\beta=5 \%, r=5 \%$ and $\sigma=0.2$. 
X. Luo and P.V. Shevchenko, Variable Annuity with GMWB: surrender or not, that is the question

\section{CONCLUSIONS}

In this paper we have developed numerical valuation of variable annuities with GMWB and surrender features under both static, dynamic (optimal) and bang-bang policyholder strategies. The results indicate that following a simple bang-bang strategy does not lead to significant reduction in the price or equivalently in the fee. We also observed that the extra value added by the surrender option very much depends on volatility and the penalty charge, among other facts such as contractual rate and maturity. At high volatility or at low penalty charge, the surrender feature adds very significant value to the GMWB contract - more than doubling in some cases; highlighting the importance of accounting for surrender feature in pricing of real products. We have assumed the policyholder will always live beyond the maturity date or there is always someone there to make optimal withdrawal decisions for the entire duration of the contract. It is not difficult to consider adding some death benefits on top of GMWB, i.e. combining GMWB with some kind of life insurance as it is done in our recent paper Luo and Shevchenko (2015b) considering both market process and death process. Further work includes admitting other stochastic risk factors such as stochastic interest rate or volatility.

\section{ACKNOWLEDGEMENT}

We gratefully acknowledge financial support by the CSIRO-Monash Superannuation Research Cluster, a collaboration among CSIRO, Monash University, Griffith University, the University of Western Australia, the University of Warwick, and stakeholders of the retirement system in the interest of better outcomes for all.

\section{REFERENCES}

Azimzadeh, Y. and P. A. Forsyth (2014). The existence of optimal bang-bang controls for gmxb contracts. Working paper of University of Waterloo.

Bacinello, A., P. Millossovich, A. Olivieri, and E. Pitacco (2011). Variable annuities: a unifying valuation approach. Insurance: Mathematics and Economics 49(1), 285-297.

Bauer, D., A. Kling, and J. Russ (2008). A universal pricing framework for guaranteed minimum benefits in variable annuities. ASTIN Bulletin 38(2), 621-651.

Bäuerle, N. and U. Rieder (2011). Markov Decision Processes with Applications to Finance. Springer, Berlin.

Chen, Z. and P. Forsyth (2008). A numerical scheme for the impulse control formulation for pricing variable annuities with a guaranteed minimum withdrawal benefit (gmwb). Numerische Mathematik 109(4), 535569.

Dai, M., K. Y. Kwok, and J. Zong (2008). Guaranteed minimum withdrawal benefit in variable annuities. Mathematical Finance 18(4), 595-611.

Huang, Y. and P. A. Forsyth (2012). Analysis of a penalty method for pricing a guaranteed minimum withdrawal benefit (GMWB). Journal of Numerical Analysis 32, 320-351.

Huang, Y. and Y. K. Kwok (2014). Analysis of optimal dynamic withdrawal policies in withdrawal guarantee products. Journal of Economic Dynamics and Control 45, 19-43.

Luo, X. and P. V. Shevchenko (2014). Fast and simple method for pricing exotic options using gausshermite quadrature on a cubic spline interpolation. Journal of Financial Engineering 1(4). DOI: $10.1142 / \mathrm{S} 2345768614500330$.

Luo, X. and P. V. Shevchenko (2015a). Fast numerical method for pricing of variable annuities with guaranteed minimum withdrawal benefit under optimal withdrawal strategy. To appear in Journal of Financial Engineering, preprint is available at http://arxiv.org/abs/1410.8609.

Luo, X. and P. V. Shevchenko (2015b). Valuation of variable annuities with guaranteed minimum withdrawal and death benefits via stochastic control optimization. Insurance: Mathematics and Economics 62, 5-15.

Milevsky, M. A. and T. S. Salisbury (2006). Financial valuation of guaranteed minimum withdrawal benefits. Insurance: Mathematics and Economics 38(1), 21-38. 